\title{
CONSENTIMIENTO INFORMADO EN ARTÍCULOS CIENTÍFICOS CON MUESTRAS PERUANAS DE ADN DEL 2001 AL 2011
}

\author{
Verónica E. Rubín de Celis ${ }^{1}$
}

\section{RESUMEN}

El consentimiento informado es una herramienta primordial para poder realizar trabajos científicos con muestras humanas. Su importancia radica en poder hacer cumplir los cuatro principios básicos de la bioética asimismo poder proteger a las personas que voluntariamente brindan su muestra de su genoma. El objetivo del trabajo es determinar el número de artículos publicados con muestras de $\mathrm{ADN}$ que no reportan el consentimiento informado entre el 2001 al 2011. Determinar las revistas en las que se publicó sin consentimiento informado y el número de muestras de ADN de individuos peruanos reportados en los artículos científicos que no reportan el consentimiento informado. Resultados: Se encontraron que los artículos publicados que no reportan el consentimiento informado fueron cinco de 1086 artículos revisados. Las revistas en las que se publicaron los artículos fueron Human Heredity, American Human Genetics. Revista Peruana de Biología, Journal Forensic Science, International Congress Series. Se encontraron 318 muestras biológicas procedentes de diferentes regiones del Perú, que en el artículo no reportaron consentimiento informado.

Se hace énfasis en la obligatoriedad del consentimiento informado en Las publicaciones.

Palabras Claves: Consentimiento informado, ADN, muestras biológicas peruanas.

\section{ABSTRACT}

Informed consent is a primary tool to perform scientific work with human samples. Its importance lies in being able to enforce the four basic principles of bioethics also to protect individuals who

Laboratorio de Genómica y Biología Molecular Evolutiva - Instituto de Ciencia y Tecnología. Universidad Ricardo Palma. 
voluntarily provide their sample genome. The aim of this work is to determine the number of articles published with DNA samples reporting no informed consent from 2001 to 2011. Determine the journals that published without informed consent and the number of DNA samples from individuals Peruvians reported in scientific articles reporting no informed consent. Results: We found that the articles published do not report the Informed Consent 1086 five articles were reviewed. The journals in which the articles were published Human Heredity, American Human Genetics. Peruvian Journal of Biology, Forensic Science Journal, International Congress Series. Found 318 biological samples from different regions of Peru, that article did not report informed consent.

Emphasis is placed on the requirement of Informed Consent in publications.

Keywords: Informed consent, DNA, biological peruvian samples

\section{INTRODUCCIÓN}

El ADN es un material que brinda la información genética que tiene un individuo, debe ser guardado y protegido en bancos genéticos en cada país de origen de la procedencia de la muestra.

Una muestra de sangre rinde mucha cantidad de ADN concentrado y para ser trabajada dicha muestra a nivel molecular debe ser diluida Con las muestras de ADN se pueden realizar infinidad de investigaciones las que pueden ser sucesivas y la muestra puede ser usada varias veces, esta muestra de ADN se obtiene a partir de cualquier fluido es decir de cualquier muestra biológica tal sea el caso de sangre, saliva, etc, y también a partir de muestras de cabello, dientes etc.

El consentimiento informado (CI) es un proceso que se expresa en un documento escrito y firmado, y que tiene que emplearse en todos los procedimientos donde los pacientes o las personas de manera voluntaria otorgan su muestra para fines de investigación o de prácticas clínicas. El consentimiento informado debe contener tres elementos fundamentales 1.- Información, 2.- Compresión y 3.- Voluntariedad.

El CI en la investigación se hace indispensable después de los horrores de la investigación realizada por los médicos nazis, en el marco de la II Gue- 
rra Mundial, en el que se experimentó con seres humanos vulnerables, y es en el primer parágrafo del código de Nuremberg donde se afirma que el consentimiento voluntario del sujeto humano es absolutamente esencial, y en los siguientes una serie de obligaciones para el investigador como: Dar a conocer la naturaleza, duración y propósito del experimento; los métodos y medios conforme a los que se llevará a cabo; los inconvenientes y riesgos que razonablemente pueden esperarse; y los efectos que para su salud o personalidad podrían derivarse de su participación en el experimento.

Por último el deber y la responsabilidad de evaluar la calidad del consentimiento corren de la cuenta de todos y cada uno de los individuos que inician o dirigen el experimento o que colaboran en él.

Por otro lado la Declaración Universal de los Derechos Humanos aprobada por las Naciones Unidas en 1948, expresa el valor humano fundamental de la protección de los derechos y bienestar de todos los sujetos humanos en la experimentación científica en la declaración Universal de los Derechos Humanos, United Nations del 2008.

Así mismo la Declaración de Helsinki elaborada por la Asociación Médica Mundial,, establece que el propósito principal de la investigación médica en seres humanos es la comprensión, causa, evolución y efectos de la enfermedad y mejoras las intervenciones preventivas, diagnosticadas y terapéuticas. Inclusive las mejores intervenciones deberán ser evaluados continuamente a través de la investigación para que sean seguras y eficaces, efectivas, accesibles y de calidad.

Esta declaración cuya primera versión el año se estableció el año 1964 en Helsinki, ha sido revisada en varias ocasiones siendo su última versión la de Seúl 2008, ha perfeccionado sus postulados llegando incluso a establecer recomendaciones en relación a la inclusión en investigación biomédica de poblaciones vulnerables.

También estableció que cualquier investigación en seres humanos antes de ser realizada deberá pasar por la revisión de los aspectos metodológicos, éticos y legales, por un Comité Institucional de ética de la investigación, quien puede observarla, aprobarla o rechazarla de plano.

Son los comités institucionales de ética en investigación biomédica, definidos como organizaciones independientes (consejo de revisión, comité 
institucional, regional, nacional o supranacional) integrados por profesionales médico, científicos y miembros no médicos/ científicos, cuya responsabilidad es asegurar la protección de los derechos, la seguridad y el bienestar de los seres humanos involucrados en una investigación y proporcionar garantía pública de esta protección a través de la revisión y aprobación, opinión favorable del protocolo de estudio, de la capacidad de los investigadores, y lo adecuado de las instalaciones, de los métodos y materiales que se usarán y documentar el consentimiento informado de los sujetos en investigación.

Después del escándalo producido en los EEUU por la denuncia de la investigación llevada a cabo en africanos americanos con sífilis en Tuskegee Alabama, la Comisión nombrada por el presidente, que trabajó de 1974 a 1978, elaboró el Reporte Belmont que establece los principios de la investigación, entre ellos el de autonomía o respeto por la autonomía de los participantes en la investigación.

El consentimiento informado es la expresión más pura del respeto a la autonomía de un sujeto. El consentimiento informado no se reduce únicamente a dar la información (aunque sea la más completa) al paciente; sino que además debe convertirse en el medio adecuado de garantizar el respeto a los derechos y la dignidad de los sujetos de investigación.

El consentimiento informado jamás debe reducirse a ser un requerimiento únicamente jurídico. Por el contrario, la obligación de obtener el consentimiento informado de los pacientes, sea en la parte clínica o investigativa, es primariamente ética, los consentimientos informados son pieza fundamental para la investigación cualquiera que ella fuera, de manera especial cuando se trata de patrimonio genético.

El Consentimiento Informado asegura que los individuos que participen en una investigación están conscientes de las condiciones en que participan, lo cual permite una decisión autónoma y el consiguiente respeto a la persona. En toda investigación biomédica que se realiza en seres humanos, el investigador debe obtener el consentimiento informado del potencial probando o en el caso de una persona incapaz de dar su consentimiento, la autorización de un representante legalmente calificado según las reglamentaciones locales. La revisión ética de un proyecto por un comité independiente garantiza al individuo que los resguardos tanto científicos como éticos serán cumplidos. 
Por último, el respeto a los sujetos inscritos debe ser resguardado mientras dure la investigación. Este respeto permite al sujeto cambiar de opinión y retirarse de la investigación sin sanción, permite mantener su privacidad y se extiende también a proporcionar el tratamiento adecuado en caso de eventos adversos. Muchos investigadores tienden a considerar que el proceso concluye al inscribirse al individuo en el estudio. Sin embargo, no es así y es necesario recalcar que este proceso se extiende mientras dure la participación del sujeto. El seguimiento continuo vela por los intereses de los probandos y permite que éstos sean informados sobre la marcha de la investigación.

Las normas internacionales CIOMS (Council for International Organizations of Medical Sciences), propuestas inicialmente en 1982, publicadas en 1991 (Epidemiología) y 1993 (Investigación biomédica) se orientaron a complementar y expandir los principios de la declaración de Helsinki en el contexto de la investigación transnacional, especialmente la que se formula en países desarrollados y se ejecuta en subdesarrollados. (Council for International Organizations of Medical Sciences).

En el presente trabajo se analizó el número de artículos publicados en el área de genética con muestras peruanas en los años 2001 a 2011. Se identificaron aquellos que no reportan la aplicación del CI a los participantes. También se determinó el número de muestras biológicas procedentes de individuos peruanos y las revistas científicas en las que se han publicado dichos artículos.

\section{METODOLOGÍA}

Se realizó una búsqueda exploratoria en las fuentes electrónicas Hinari, Pubmed, Scielo y Google Académico, de aquellas referencias bibliográficas en las que se hayan usado muestras biológicas peruanas, con posibilidad de obtener ADN humano para realizar investigaciones y no se haya sido reportado en la publicación el uso del consentimiento informado, entre los años 2001 al 2011.

Se empleó el programa estadístico SPPS versión 20 para analizar los artículos que reportaban muestras de ADN humano peruano sin el reporte del consentimiento informado. Se consideraron las siguientes variables: el número de artículos sin reporte de consentimiento informado, el número de individuos reportados en cada publicación, las revista de publicación, 
la fuente de financiamiento, el año de la publicación, la institución ejecutora.

\section{RESULTADOS}

De los 1086 artículos revisados que cumplían los criterios de búsqueda, solo 5 de ellos no reportan consentimiento informado para el empleo de ADN humano procedente de individuos peruanos en la publicación, lo que constituye el $0,46 \%$ del total de los artículos.

Tabla 1. Artículos encontrados en la revisión. (Anexo)

En esta tabla se consignan los diez artículos en los que se reporta investigación con muestras biológicas humanas provenientes de pobladores peruanos, por revista, año de publicación, número de muestras tomadas.

En la figura $\mathbf{N}^{\circ} \mathbf{1}$ se muestra el número de muestras biológicas en las publicaciones evaluadas en rosado.

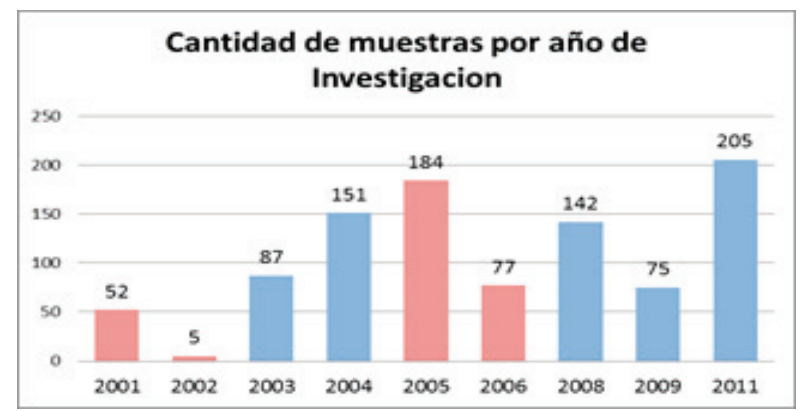

En relación a la cantidad de muestras biológicas empleadas en cada año por publicación se resalta que en el año 2005 se tomó el mayor número de muestras, de la misma manera se presentan en el 2011, teniendo un total de 205 muestras.

Se observa en color rojo que en los años 2001, 2002, 2005 y 2008 se reportaron artículos en las revistas que no figuraban el consentimiento informado de la toma de muestras. Es importante señalar que en el año 2005 se presentan 184 muestras en los artículos que reportan consentimiento informado. (Rupert et al. 2003, Sandoval et al. 2004, Abigail et al. 2008, 
Abigail et al. 2009, Córdova et al. 2011)

Es importante mencionar que cuando se comparan las publicaciones de los artículos realizadas por investigadores nacionales que reportan consentimiento informado tenemos 151 muestras, mientras que sin el reporte del consentimiento informado tenemos 184 muestras.

Todos los investigadores extranjeros que reportaron en sus artículos el consentimiento informado emplearon 367 muestras.

Siendo que cuando la publicación fue realizada entre investigadores nacionales y extranjeros 142 muestras son reportadas en los artículos con el consentimiento informado y 134 sin consentimiento informado.

\section{Relación número de muestras y \\ consentimiento informado}

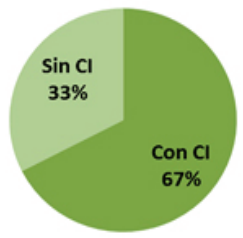

Fig $\mathbf{N}^{\circ} 2$ Artículos publicados con reporte del consentimiento informado: $33 \%$ mientras los artículos publicados con reporte del consentimiento informado presenta un $67 \%$ del total de los artículos.

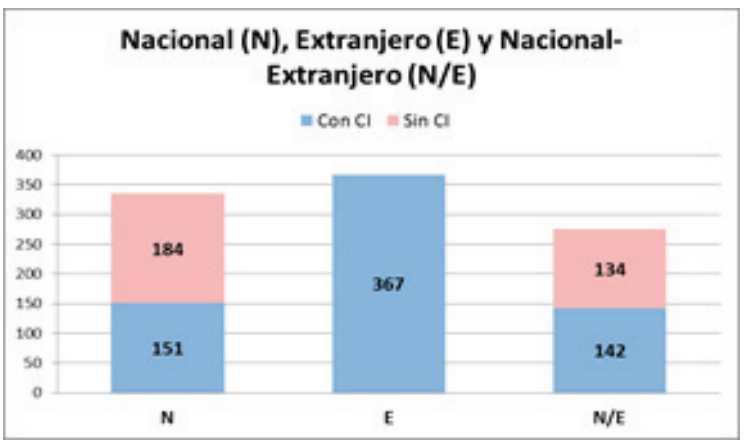

Fig No 3. Relación de publicaciones de investigadores nacionales y extranjeros con consentimiento informado y sin consentimiento informado. 


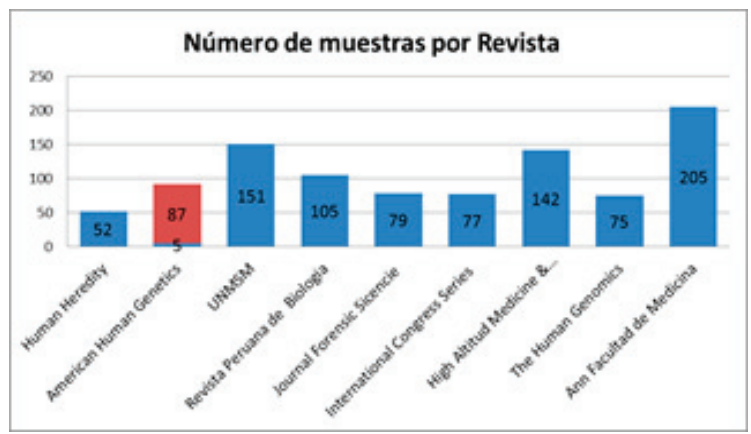

Fig $\mathbf{N}^{\circ}$ 4. Relación de revistas relacionadas con el número de muestras empleadas en los artículos y la revista donde se publicó.

\section{DISCUSIÓN}

El consentimiento informado es procedimiento importante que se expresa en un documento escrito y firmado, y que tiene que emplearse en todos los procedimientos donde los pacientes o las personas de manera voluntaria otorgan su muestra para fines de investigación. Se encontraron 5 artículos que no reportan el consentimiento informado, lo que a pesar de ser un pequeño porcentaje de $33 \%$, del total de los artículos publicados, representan un alto número de participantes peruanos, que proporcionaron muestras biológicas probablemente sin haber sido adecuadamente informados.

Existen antecedentes históricos como el caso de la investigación con el grupo étnico de los Yanomami en Brasil, en el que cinco universidades norteamericanas tras un pedido de las autoridades Brasileras, aceptaron devolver miles de muestras de sangre extraídas en el año 1967 a indígenas de esa tribu amazónica, en lo que constituyó uno de los casos más controvertidos de la denominada "biopiratería" mundial.

El genetista Francisco Salzano, negó que el accionar con los Yanomamis en Venezuela y Brasil haya sido "biopiratería" y relativizó la cuestión ética sobre el consentimiento para la extracción de sangre.

Uno de los objetivos era hacer el mapa del ADN de los nativos yanomamis para evaluar el aislamiento geográfico y para hacer el trayecto de la migración humana hacia el continente americano, en la Era del Hielo. 
Salzano manifestó sobre la extracción de sangre sin consentimiento de los indígenas que "el consentimiento informado es relativo en cualquier grupo marginal, incluso urbano. No es posible esperar que esos grupos entiendan lo que ciencia planea hacer con el ADN de ellos". Matriz del Sur (2010)

Es importante resaltar que las revistas con este tipo de publicación presentan un comité de ética. Debemos de especificar que la mayoría de trabajos realizados con muestra de $\mathrm{ADN}$ peruano humano que no reportan el consentimiento informado son de científicos nacionales como se ha descrito en los resultados. (Iannacone et al. 2005, Carbajal-caballero et al. 2005)

Sin embargo tenemos otros trabajo publicados en revistas extranjeras con muestra de ADN peruano, donde es trabajo es realizado por investigadores nacionales y extranjeros que no reportan en su publicación, el consentimiento informado.(Novo 2007, Council for International Organizations of Medical Sciences (1993).

En los artículos publicados no se menciona dónde se va a preserva la muestra sobrante de la colecta y en muchos artículos no se menciona la cantidad de muestra colectada (Rodríguez-Delfín et al.2001) (Silva WA Jr. et al. 2002 ) (Rupert et al.2008, Sandoval et al.2004, Iannacone et al. 2005, Carbajal-caballero et al. 2005)

Encontramos artículos donde los autores extranjeros reportan el consentimiento informado y están conscientes de la importancia del mismo como se puede apreciar dentro de nuestros resultados. (Rupert et al. 2003, Abigail et al .2008, Abigail et al. 2009)

Se encontró que en uno de los artículos se coloca el término el "adecuado consentimiento" teniendo en la muestra de la población niños de colegios entre 10-18. Creemos que el término adecuado que debería colocarse es consentimiento informado de los padres o asentimiento de los niños (Sandoval et al. 2004).

1. Estudiaron la variabilidad genética de la población Quechua del Perú. Ellos emplearon 52 individuos procedentes de Pasco en número de 28 y 24 de lima. Los individuos tuvieron por lo menos un apellido quechua para la selección. El artículo no reportó el consentimiento informado de las muestra empleadas en esta investigación (Rodríguez-Delfín et al. 2001) 
2. Estudiaron la diversidad mitocondrial del genoma de nativos americanos apoya una sola entrada de una sola población fundadora en América. Analizaron el adn mitocondrial a través de las secuencias de la región controladora. Estudiaron el genoma mitocondrial de 40 individuos nativos americanos de la literatura. Tomaron la muestra de treinta nativos americanos de diferente procedencia que podrían tener los cuatro haplogrupos de los nativos americanos y colectaron muestra de cinco quechuas procedentes de Perú que no reportaron en el artículo consentimiento informado. (Araujo-Silva Jr. et al. 2002).

3. Analizaron los haplotipos del cromosoma Y a través del Powerplex y doce STRs en una muestra de la población peruana. Reportaron muestras de las regiones políticas de Cajamarca, la libertad, Lambayeque, Piura, Tumbes, Amazonas, Iquitos, Ucayali, Ancash, Cerro de Pasco, Huancavelica, Huánuco, Junín, Lima, Ica, Apurímac, Ayacucho, Cusco, Puno y Tacna. Estas muestras fueron de 79 varones con linaje paternos y fueron obtenidas de los familiares del casos de mesa redonda, de los exámenes de paternidad y casos de identificación genética, en el artículo no se reportó el consentimiento informado. (Innacone et al. 2005)

4. Evaluaron los loci del cromosoma Y especifico del DYS287, DYS199, DYS390. El total de la muestra fueron 105 individuos varones de cuatro comunidades nativas, las muestras fueron seleccionadas por el tipo de apellidos oriundos de la zona: Aguaruna, Yamayakat, Santiago de chuco, Trujillo, Dentro de la metodología, no reporta la presencia del consentimiento informado.(Carbajal-Caballero et al. 2005 )

5. Estudiaron y establecieron 16 Y-STR (DYS19, DYS385, DYS3891/ ii,, DYS390, DYS391, DYS392, DYS 393, DYS437, DYS438, DYS439, DYS460, DYS461, GATA-A10,GATA-H4 y DYS635 en una población de 77 hombres del Perú, siendo que encontraron 76 haplotipos diferentes, setenta y cinco haplotipos fueron únicos y solo uno fue detectado en dos hombres. La diversidad haplotípica fue $0,9997+-0,00022$. Construyeron haplotipos altamente informativos que permitieron la discriminación de $97.4 \%$ de las muestra usadas en el estudio. Lo que representa una poderosa herramienta individual de identificación para la prueba de paternidad en medicina forense. 
La publicación no reporta que las muestra tengan el consentimiento informado ( Builes J. et al. 2006)

\section{CONCLUSIONES}

1. El número de artículos de muestras de $\mathrm{ADN}$ peruano que no reportan el consentimiento informado; son cinco en los años 2001 al 2011, constituyendo solo un pequeño porcentaje del total de publicaciones.

2. El número de muestras biológicas con ADN humano peruano empleadas por investigadores sin consentimiento informado ascendió a 316 muestras entre hombres, mujeres y niños.

3. Las revistas internacionales que publicaron los artículos sin el reporte del consentimiento informado fueron: International Congress Series, Journal Forensic Science, Human Heredity, American Human Genetics siendo la revista nacional la Revista Peruana de Biología.

\section{RECOMENDACIONES}

1. Todas las revistas deberían considerar como obligatoria la aprobación de los trabajos por un Comité de bioética la Investigación en las instituciones donde se ejecute.

\section{REFERENCIAS BIBLIOGRÁFICAS}

1. Novo F. (2007). Genética humana. Ed Pearson. Prentice Hall.pa $1-290$

2. Garzon A.( 2009). Aspectos bioéticos da informação subministradas na pesquisa biomédica com uma população vulnerável. Rev. Latinoam. bioet. Vol $9 \mathrm{~N}^{\circ} 2$. Bogotá.

3. Quiroz E. (2010). ¿Por qué auditar a los comités de ética de investigación. Rev. Perú. Med. Exp. Salud Pública. 27 (3) 443-48.

4. Helsinki Declaration Available (2013). Disponible en: http://www. wma.net/es/30publications/10policies/b3. Acceso el 8 de agosto 2013.

5. Nuremberg Code. Available at: http//www.hhs.gov/ohrp/archive/index $/ \mathrm{html}$. Acceso el 8 de agosto 2013.

6. Informe Belmont www.pcb.ub.edu/bioeticaidret/archivos/norm/InformeBelmont.pdf'de PYGÉ PARA - [1979 . Acceso el 8 de agosto 2013 
7. Council for International Organizations of Medical Sciences. International Ethical Guidelines for Biomedical Research Involving $\mathrm{Hu}-$ man Subjects. Ginebra: CIOMS, 1993

8. Marianne Gaudlitz H. (2008). Reflexiones sobre los principios éticos en investigación biomédica en seres humanos. Rev. Chil. Enf. Respir. $\quad 24: 138-142 \mathrm{http}: / / w w w . s c i e l o . c l / s c i e l o . p h p ? p i d=S 0717-$ $73482008000200008 \&$ script $=$ sci_arttext

9. Rodríguez-Delfín, Rubín de Celis V and Marco A. Zago M. (2001). Genetic Diversity in an Andean Population from Peru and Regional Migration Patterns of Amerindians in South America: Data from Amerindians in south America Y Chromosome and Mitochondrial DNA. Hum Hered 2001; 51:97-106

10. Silva WA Jr, Bonatto SL, Holanda AJ, Ribeiro-Dos-Santos AK, Paixão BM, Goldman GH, Abe-Sandes K, Rodríguez- Delfín L, Barbosa M, Paçó-Larson ML, Petzl-Erler ML, Valente V, Santos SE, and Zago MA, (2002). Mitochondrial genome diversity of Native Americans supports a single early entry of founder populations into America. Am. J, Hum Genet. Jul; 71(1):187-92.

11. Rupert, J. L, Kidd, K. K, L. E. Norman, M. V. Monsalve, P. W. Hochachka and D. V. Devine (2003). Genetic Polymorphisms in the Renin-Angiotensin System in High-Altitude and Low-Altitude Native American Populations .Annals of Human Genetics 67,17-25

12. Sandoval J, Bedsabé Delgado, Luis Rivas, Bertha Bonilla, Daniel Nugent y Ricardo Fujita. (2004). Variantes del ADNmt en isleños del lago Titicaca: máxima frecuencia del haplotipo B1 y evidencia de efecto fundador. Rev. peru. biol. 11(2): 161-168

13. Iannacone G, Raúl tito, Paul Wenceslao López, María Elena Medina y Beatriz Lizárraga. (2005). Journal Forensic Science. Vol 50 N 1- 4.

14. Carbajal-Caballero N, Susy Núñez, Milenka Narvaiza, Carlos Aguirre, Carlos Villanueva, Juan Muro y Luis Rodríguez-Delfín. (2005). Polimorfismos del cromosoma $\mathrm{Y}$ humano en poblaciones de la región norte del Perú Rev. Peru. biol. 12(3): 341- 348.

15. Juan José Builes, J Hau, JJ. Rodríguez, A.E Montoya, F. Izarra, O. Ochoa. L. Perez. M. de Pancorbo, L. Gusmao, y M.L J Bravo. (2006). 
Peruvian population study with 16 Y_STR loci. International Congress Series 1288 : 216-218.

16. Abigail W. Bigham, Melisa Kiyamu, Fabiola León-Velarde, Esteban J. Parra, Maria Rivera-Ch, Mark D. Shriver, and Tom D. Brutsaert (2008). Angiotensin-Converting Enzyme Genotype and Arterial Oxygen Saturation at High Altitude in Peruvian Quechua. HIGH ALTITUDE MEDICINE \& BIOLOGY Volume 9, Number 2

17. Abigail W. Bigham, Xianyun Mao, Rui Mei, Tom Brutsaert, Megan J. Wilson, Colleen Glyde Julian, Esteban J. Parra,Joshua M. Akey, Lorna G. Moore and Mark D. Shrive. (2009). Identifying positive selection candidate loci for high-altitude adaptation in Andean populations. HUMAN GENOMICS. VOL 4. NO. 2. 79-90 2009

18. Córdova J, Ricardo Fujita, José Sandoval, Jaime Descailleaux, Margarita Velásquez, Calen Távara, y Claudia Barletta. (2011). Divergencia genética en poblaciones peruanas detectada a partir delas frecuencias haplotípicas del mtDNA y del gen nuclear MBL An Fac med. 2011;72(1):51-9

19. Matriz del Sur (2010). Biopiratería: EEUU acepta devolver sangre extraída en 1967 a indígenas Yanomami de Brasil y Venezuela Matriz del Sur - www.aporrea.org 10/05/10 - www.aporrea.org/internacionales/n156934.html0.-

20. Organización de las Naciones Unidas. (2008). Declaración Universal de los Derechos Humanos, United Nations. Recuperada en Junio 15, 2009, del sitio Web tema: Portal de Recursos Educativos Abiertos (REA) en http://www.temoa.info/es/node/19618 


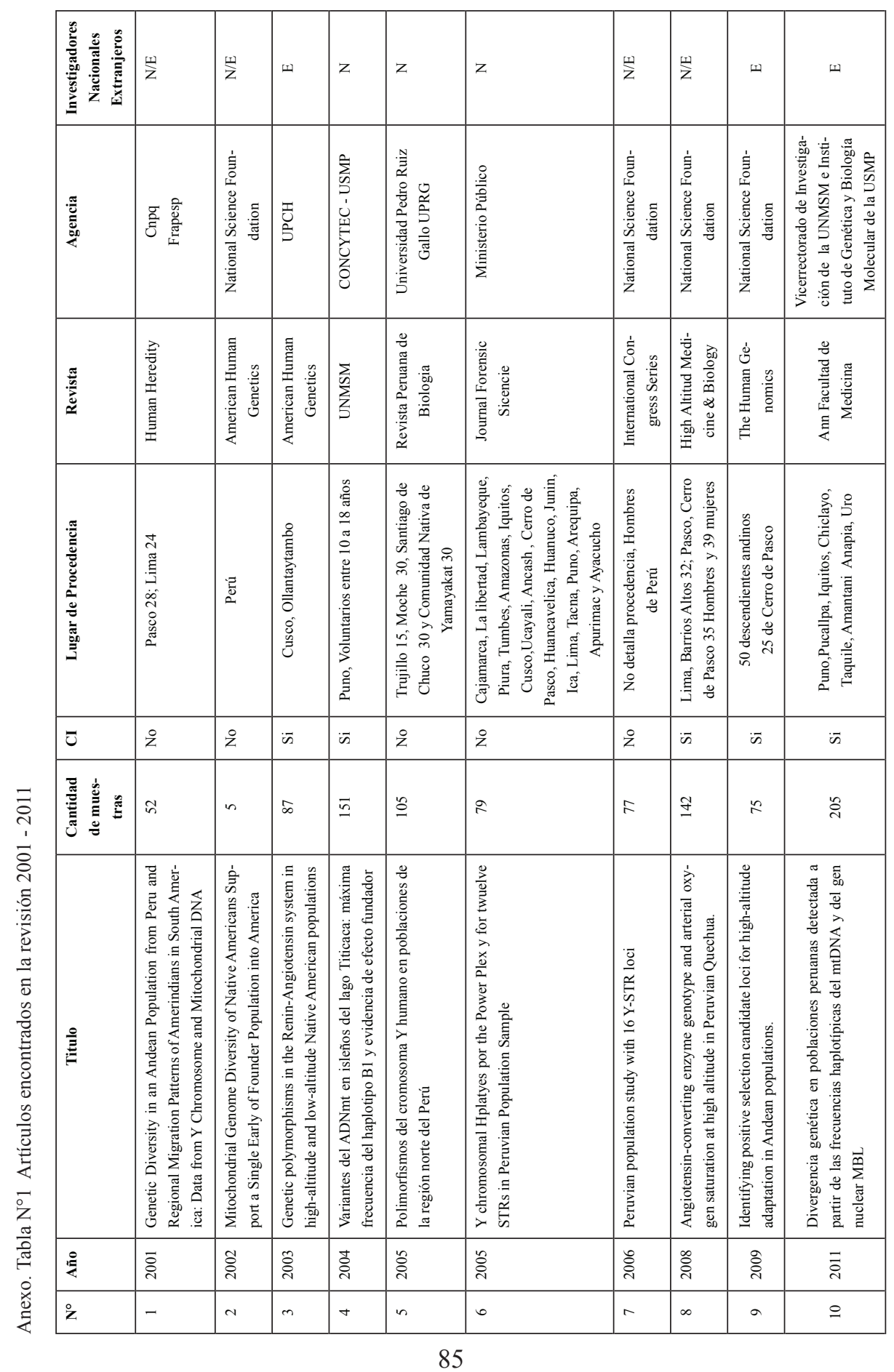

